\title{
Multi-step inhibition explains HIV-1 protease inhibitor pharmacodynamics and resistance
}

\author{
S. Alireza Rabi, ${ }^{1}$ Gregory M. Laird, ${ }^{1}$ Christine M. Durand,, ${ }^{1}$ Sarah Laskey, ${ }^{1}$ Liang Shan, ${ }^{1}$ \\ Justin R. Bailey, ${ }^{1}$ Stanley Chioma, ${ }^{2}$ Richard D. Moore, ${ }^{1}$ and Robert F. Siliciano ${ }^{1,2}$ \\ 1Department of Medicine, Johns Hopkins University School of Medicine, Baltimore, Maryland, USA. \\ ${ }^{2}$ Howard Hughes Medical Institute, Baltimore, Maryland, USA.
}

\begin{abstract}
HIV-1 protease inhibitors (PIs) are among the most effective antiretroviral drugs. They are characterized by highly cooperative dose-response curves that are not explained by current pharmacodynamic theory. An unresolved problem affecting the clinical use of PIs is that patients who fail PI-containing regimens often have virus that lacks protease mutations, in apparent violation of fundamental evolutionary theory. Here, we show that these unresolved issues can be explained through analysis of the effects of PIs on distinct steps in the viral life cycle. We found that PIs do not affect virion release from infected cells but block entry, reverse transcription, and post-reverse transcription steps. The overall dose-response curves could be reconstructed by combining the curves for each step using the Bliss independence principle, showing that independent inhibition of multiple distinct steps in the life cycle generates the highly cooperative dose-response curves that make these drugs uniquely effective. Approximately half of the inhibitory potential of PIs is manifest at the entry step, likely reflecting interactions between the uncleaved Gag and the cytoplasmic tail (CT) of the Env protein. Sequence changes in the CT alone, which are ignored in current clinical tests for PI resistance, conferred PI resistance, providing an explanation for PI failure without resistance.
\end{abstract}

\section{Introduction}

HIV-1 protease inhibitors (PIs) have played a critical role in the success of highly active antiretroviral therapy (HAART) (1-3). PIs are the key drugs in 2 of the 4 recommended initial HAART regimens and are also extremely important in salvage therapy for patients who fail initial regimens (1). Among all HIV-1 drugs, PIs have the highest intrinsic antiviral activity $(2,4)$. PIs are the only antiretroviral drugs that have been successfully used in monotherapy (5). The high antiviral activity of this class results in large part from steep, highly cooperative dose-response curves $(2,4)$, the molecular basis of which is not fully understood (6).

The PIs are substrate or transition state analogues that inhibit the activity of HIV-1 protease. This enzyme cleaves viral polyproteins during virus maturation (7). Among the protein products of the HIV-1 genome are 3 polyproteins: the envelope (Env) precursor protein (gp160), the Gag precursor protein (Pr55 Gag), and the Gag-Pol precursor protein $\left(\operatorname{Pr} 160^{\mathrm{Gag}-\mathrm{Pol}}\right)$. A cellular protease cleaves gp160 into the surface and transmembrane subunits, gp120 and gp41, respectively (8). These subunits remain associated, and trimers of gp120/gp41 complexes constitute the surface spikes that mediate viral entry. In contrast, the Gag and Gag-Pol polyproteins are each cleaved into multiple mature virion proteins by HIV-1 protease. The cleavages carried out by HIV-1 protease occur within the nascent virus particle and produce mature virions capable of infecting new cells.

While the interaction of PIs with the target enzyme is well understood at the structural and biochemical level (9-12), it remains unclear where in the virus life cycle the inhibition of virus maturation becomes manifest. Virus maturation is generally considered

Authorship note: S. Alireza Rabi and Gregory M. Laird contributed equally to this work.

Conflict of interest: The authors have declared that no conflict of interest exists. Citation for this article: J Clin Invest. 2013;123(9):3848-3860. doi:10.1172/JCI67399. to be important for early postentry steps including uncoating and reverse transcription (13-15). Both the RT and integrase (IN) enzymes are generated from $\operatorname{Pr} 160^{\mathrm{Gag}-\mathrm{Pol}}$ by cleavages carried out by HIV-1 protease. However, inhibition of the proteolytic cleavages necessary for maturation could in principle affect other steps as well. Studies of mutant viruses incapable of completing the necessary proteolytic cleavages suggest that immature particles are defective in entry (16-18). Interactions between the cytoplasmic tail (CT) of gp41 and uncleaved Pr55 Gag appear to inhibit the fusion of immature particles. Despite the importance of PIs in HIV-1 treatment, the precise step or steps in the virus life cycle blocked by these drugs under clinical conditions have not been clearly defined.

Understanding where the PIs act in the virus life cycle is also important for understanding the resistance that arises in some patients on PI-based regimens. Resistance to PIs can occur through mutations in the protease gene (19), but the majority of patients failing PI-containing regimens do so without mutations in protease (20-23). This interesting and unexpected observation appears to violate the basic evolutionary tenets that govern other types of HIV-1 drug resistance. It also poses an important clinical dilemma - should treatment be changed for patients with detectable viremia but no mutations in protease? One possibility is that standard assays for resistance ignore parts of the HIV-1 genome that may contain mutations conferring resistance to PIs.

To understand the molecular mechanisms responsible for the high antiviral activity of PIs and the unusual features of resistance to these drugs, we carried out a detailed dissection of the points in the life cycle affected by inhibition of protease. We experimentally isolated each relevant step of the life cycle and measured the dose-response curves of PIs for each isolated step. We then reconstructed the overall dose-response curve by combining the curves from each step. This analysis provided a mechanistic explanation 
A
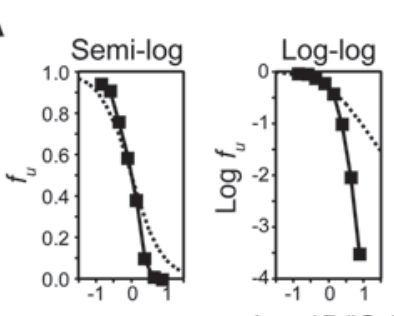

$\log \left(D / / C_{50}\right)$

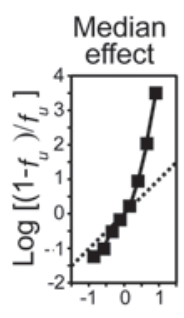

B

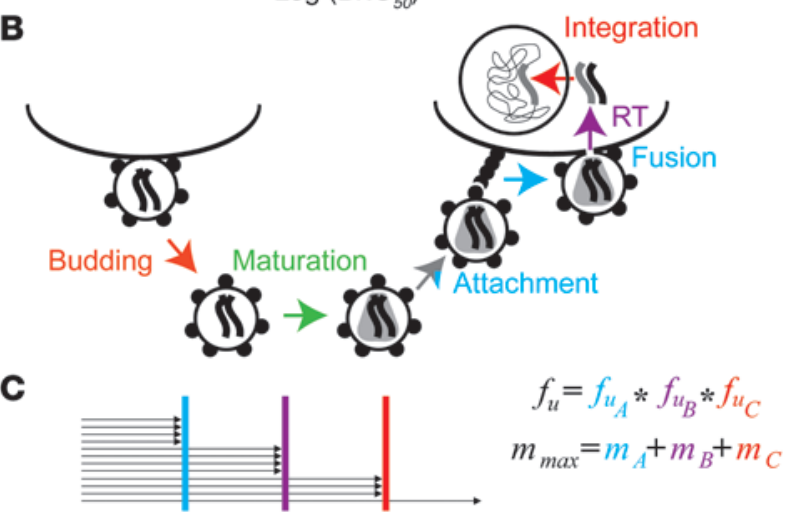

\section{Figure 1}

PI pharmacodynamics. (A) Representations of the dose-response curves for the PI ATV. Primary CD4+ ${ }^{+}$cells were infected with viruses generated in the presence of various ATV concentrations, and $f_{u}$ was measured as previously described (2). Left panel: conventional semi$\log$ dose-response curve in which $f_{u}$ is plotted against $\log D$ (normalized by $I C_{50}$ ). Conventional plots obscure the differences between the ATV curve and the curve for a hypothetical drug with the same $I C_{50}$ and an $m$ value of 1 (dotted line). Middle panel: log-log of the doseresponse curve. Right panel: median effect plot, $\log \left[\left(1-f_{u}\right) / f_{u}\right]$ vs. log $D / / C_{50}$. This plot, based on Equation 2, linearizes most dose-response curves, resulting in lines whose slopes are equal to the slope parameter $m$ in Equations 1 and 2. This plot illustrates the steep slope and upward inflection of PI dose-response curves. (B) PIs may inhibit multiple steps in the life cycle. Pls block maturation of the virus particle (green arrow). Since maturation begins concomitantly with budding, an effect on budding is possible. Viruses that fail to mature due to the action of PIs could be blocked at downstream steps including entry, reverse transcription, and integration. (C) If PIs block multiple steps, then Bliss independence predicts that the fraction of successful infection events is the product of the fraction of viruses that pass each block. The maximal slope of the overall dose-response curve is the sum of the slopes of the doseresponse curves for each step (Supplemental Appendix 1). for the unique pharmacodynamics and exceptional efficacy of PIs. It also provided a potential explanation for PI resistance in the absence of mutations in protease. In light of these findings, we advocate for a reevaluation of current clinical assays for resistance to these important drugs.

\section{Results}

Rationale. Using single-round infectivity assays, we have previously demonstrated unexpected complexity in the dose-response curves for antiretroviral drugs $(2,4)$. At least 2 drug-specific parameters are necessary to fully describe these curves: the concentration of drug producing $50 \%$ inhibition $\left(I C_{50}\right)$ and the slope $(m)$, which describes the steepness of the curve. The fraction of infection events unaffected $\left(f_{u}\right)$ by the drug at a concentration $D$ is given by the median effect equations (24):

$$
f_{u}=\frac{1}{1+\left(D / I C_{50}\right)^{m}}
$$

\section{(Equation 1)}

or

$$
\begin{aligned}
& \log \left[\left(1-f_{U}\right) / f_{u}\right]=m \log \left[\frac{D}{I_{50}}\right] \\
& \text { (Equation 2) }
\end{aligned}
$$

Standard semi-log dose-response curves plot $f_{u}$ vs. $\log D$. In comparing multiple drugs, it is useful to normalize the drug concentration by the $I C_{50}$. Plots of this kind obscure the importance of the slope parameter (Figure 1A). The dramatic effects of the slope parameter are much more evident in log-log plots or plots based on the median effect equation (Equation 2). Using this equation, dose-response curves that follow the standard Hill or sigmoi- dal $E_{\max }$ models can be linearized. The $m$ values can be obtained directly from the slopes of the resulting lines. Generally, $m$ values greater than 1 reflect cooperative interactions $(6,25,26)$. The clinical significance of the slope lies in the fact that with high slope values, small increases in drug concentration result in large increases in inhibition, as shown in log-log and median effect plots (Figure 1A). In this context, the dose-response curves of PIs have 2 important features: first, the curves are steep, with an overall slope of greater than 1 . Second, the median effect plots are nonlinear, with a pronounced upward inflection (Figure 1A; median effect plot). This upward inflection is not explained by any standard pharmacodynamic model. As a consequence of these 2 features, small increases in PI concentration can result in dramatically increased inhibition.

We hypothesized that these unique features of PI dose-response curves reflect the fact that virus particles generated in the presence of PIs can be blocked at multiple downstream steps in the virus life cycle (Figure 1B). Generally, failure to complete a step in the virus life cycle precludes all subsequent steps. Although PIs block the maturation of the virus particle, the subsequent step in the life cycle at which the failure in maturation becomes manifest is not completely understood, and it is possible that multiple downstream steps are affected (Figure 1, B and C). Viruses that successfully complete the first downstream step (step A) could still be blocked at a subsequent step. The inhibition at the second step (step B) would be independent because only viruses that were not inhibited at step A can be blocked at step B. In this situation, the Bliss independence model can be used to compute the combined effects (27). As shown in Figure 1C, if $f_{u A}, f_{u B}$, and $f_{u C}$ are the fractions of viruses that are blocked at steps A, B, and C of the life cycle, then the fraction of viruses that can complete the entire life cycle $\left(f_{u}\right)$ is as follows:

$$
f_{u}=f_{U A} \times f_{U B} \times f_{U C}
$$

(Equation 3) 
Each inhibited step has its own dose-response curve, which can be described with $I C_{50}$ and $m$ values. Thus,

$$
f_{u}=\frac{1}{1+\left(D / I C_{50_{A}}\right)^{m_{A}}} \times \frac{1}{1+\left(D / I C_{50_{B}}\right)^{m_{B}}} \times \frac{1}{1+\left(D / I C_{50_{C}}\right)^{m_{C}}}
$$

This equation predicts steep dose-response curves that inflect upward to give a maximal slope that can be shown to be equal to the sum of the slopes of the dose-response curves at steps A, B, and C (Supplemental Appendix 1; supplemental material available online with this article; doi:10.1172/JCI67399DS1). Thus the steep dose-response curves for PIs could be explained by the fact that a single drug blocks multiple downstream steps in the life cycle.

To test this hypothesis, we developed experimental methods to isolate each potential step at which virions generated in the presence of PIs could be blocked. Using these assays, we measured the $I_{50}$ and $m$ for each step of the life cycle inhibited by PIs and reconstructed the overall dose-response curves using the Bliss independence model $(4,27)$.

PIs do not block budding. The processes of viral budding and maturation occur concomitantly (28). Although the Gag polyprotein is necessary and sufficient for budding (29-31), it is conceivable that PIs could affect this step. For example, several PIs inhibit proteasome function (32-34) and some proteasome inhibitors, such as epoxomicin, block retroviral budding $(35,36)$.

To determine whether inhibition of virion maturation affects budding, we developed an assay for the detection of virus particle released from virus-producing cells. Synchronous analysis of the budding step can be carried out using cells transfected with proviral constructs. However, in this situation, analysis of virus particle release by RT-PCR is complicated by the presence of plasmid DNA even after DNase treatment. Therefore, we developed an RT-PCR method that allowed specific quantitation of genomic viral RNA in virions released from transfected cells even in the presence of micromolar concentrations of contaminating HIV-1 DNA. 293T cells were cotransfected with a plasmid carrying the HIV-1 NL4-3 provirus with GFP in the $e n v$ open reading frame (ORF) (NL4-3 $\Delta$ Env) and a vector expressing an X4-tropic Env (from NL4-3). After 48 hours of incubation in the presence of PIs, supernatants were treated with a genetically modified bacterial endonuclease (Benzonase) that degrades all forms of DNA and RNA, including single- and double-stranded forms and circular and linear forms. This enzyme can thus degrade free, extravirion RNA, plasmid DNA, and proviral DNA. Intravirion viral RNA was then measured by quantitative RT-PCR. The 3 ' primer consisted of $25 \mathrm{dTs}$ followed by 5 nucleotides (GAAGC) complementary to the last 5 nucleotides in the R region of the LTR. This PCR strategy allows specific amplification of HIV-1 mRNAs without detection of proviral or plasmid DNA (see Methods). The 5' primer anneals to a highly conserved region in the $\mathrm{U} 3$ region LTR. Unlike other viral quantification assays that use RT-PCR, this assay is unaffected by contaminating proviral DNA or plasmid DNA since only polyadenylated mRNA can be detected (Supplemental Figure 1). In addition, pretreatment of supernatant with a nuclease ensures that only intravirion RNA will be measured and not mRNA released from dead cells (Supplemental Figure 2). Unlike ELISA-based techniques that measure the amount of HIV-1 Gag p24 antigen in the supernatant, the quantitative RT-PCR-based (RT-qPCR-based) assay described here is inde- pendent of the maturation state of the virion and detects only particles containing viral RNA.

Using this assay, we demonstrated that virion release from virusproducing cells is not affected by PIs. Figure 2 shows results for the 3 most widely used PIs: atazanavir (ATV), darunavir (DRV), and lopinavir (LPV). The log-log dose-response curves show no decrease in the release of virus particles as the PI concentration increases. This is true even at concentrations up to 100 -fold above the $I C_{50}$ for inhibition of infectivity and even for PIs reported to block the cellular proteosomal activity (refs. 32-34 and Supplemental Figure 3).

Viruses produced in the presence of PIs show defects in entry. To determine whether PIs inhibit entry, we utilized a previously described assay for viral entry based on fluorescence resonance energy transfer (FRET) (37). Briefly, the enzyme $\beta$-lactamase was incorporated into virus particles as a fusion protein with the HIV-1 accessory protein Vpr (BLAM-Vpr). Upon entry, BLAM-Vpr cleaves a fluorescent dye that is preloaded into the target cells, resulting in a shift in the emission spectrum detectable with flow cytometry. Importantly, we first showed that PI treatment does not affect the activity of the BLAM-Vpr fusion protein (Supplemental Figure 4). To assess the effects of PIs on HIV-1 entry, HEK 293 T cells were cotransfected with NL4$3 \Delta$ Env, an expression vector for an X4-tropic Env, and a plasmid expressing BLAM-Vpr. Transfected cells were then distributed into 96-well plates, and PIs were added. After 48 hours of incubation in the presence of PIs, virus-containing supernatants were collected and used to infect primary $\mathrm{CD} 4^{+} \mathrm{T}$ lymphoblasts. Entry was assessed by flow cytometry as a green-to-blue shift in individual cells, as described (37).

All PIs tested produced a dose-dependent inhibition of the entry of pseudoviruses carrying WT HIV-1 Env. As shown in the $\log -\log$ dose-response curves in Figure 2, the fraction of entry events unaffected by the drug $\left(f_{u}\right)$ decreased with increasing drug concentration for ATV, DRV, and LPV. This entry inhibition was dependent on the nature of the envelope protein used. Pseudoviruses generated using the vesicular stomatitis virus $G$ protein (VSV-G) rather than HIV-1 Env were not inhibited at entry by PIs. In addition, truncation of the gp41 CT largely relieved the PImediated inhibition of entry (Figure 2). These results are consistent with previous studies showing that viruses with mutations in the Gag cleavage sites recover their ability to fuse if pseudotyped with VSV-G or an HIV-1 envelope that has a truncation of the gp41 CT $(17,18)$. In these viruses, interactions between the gp41 $\mathrm{CT}$ and the matrix (MA) component of uncleaved Gag precursor protein $\left(\operatorname{Pr} 55^{\mathrm{Gag}}\right)$ that may inhibit entry cannot take place. In subsequent experiments, we used virus particles pseudotyped with VSV-G or with an HIV-1 Env with a truncated gp41 CT to bypass the entry inhibition of PIs and examine the effects of these drugs on subsequent steps in the life cycle.

Viruses produced in the presence of PIs are blocked at multiple steps in the life cycle. Because the BLAM-Vpr assay detects only effects on the entry step, the above results clearly demonstrate that PIs inhibit HIV-1 entry. To compare the effects of PIs on different steps in the life cycle, we used median effect plots (Figure 3). Unlike the upwardly inflected dose-response curves for the overall inhibition of infectivity (Figure 1A, median effect plot, and refs. 2, 4), the inhibition of entry resulting from PI treatment of virus-producing cells gave linear dose-response curves throughout the dynamic range of the assay (up to 1.5 logs above the $I C_{50}$ ). By extrapolating these straight lines into the clinical concentration range, we estimated that at 

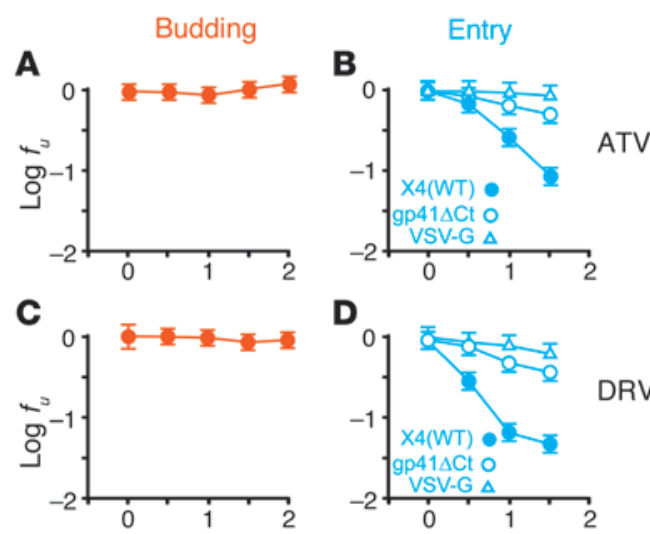

E

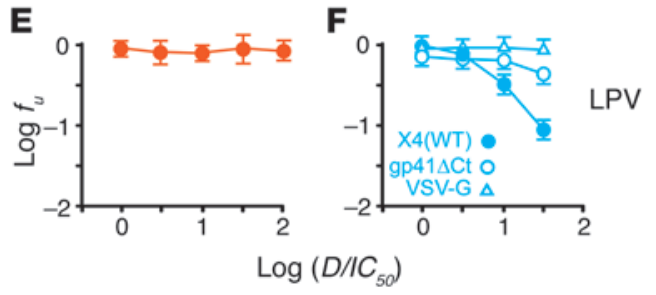

peak plasma concentrations $\left(C_{\max }\right), \mathrm{ATV}, \mathrm{DRV}$, and LPV produce 2.8, 5.0 and 2.7 logs of inhibition at the entry step, respectively.

The inhibition of entry by PIs is not complete. For some virions generated in the presence of PIs, entry occurs and downstream steps in the viral life cycle can take place. To evaluate the effect of PIs on reverse transcription, we infected $\mathrm{CD}^{+} \mathrm{T}$ lymphoblasts with HIV-1 pseudoviruses carrying a truncated form of gp41. As shown in Figure 2, PIs have only a minimal inhibitory effect on the entry of viruses with a truncated gp41 CT at concentrations up to 10 -fold above the $I C_{50}$. This allowed us to isolate postentry effects. Pseudoviruses were made by cotransfecting $293 \mathrm{~T}$ cells with NL4-3 $\Delta$ Env and an expression vector for an X4-tropic Env truncated at L753 of the gp41 CT (HXB2 coordinates). At 48 hours after transfection, virus-containing supernatants were collected and used to infect primary $\mathrm{CD} 4^{+} \mathrm{T}$ cells from healthy donors. At 36 hours after infection, $\mathrm{CD}^{+} \mathrm{T}$

\section{Figure 3}

Median effect plots illustrating the effects of the PIs ATV, DRV, and LPV on HIV-1 entry, reverse transcription, all postentry events, and overall infectivity. (A, E, and I) Effect of PIs on viral entry. The doseresponse curves of Pls at the entry step from Figure 2 were linearized by plotting $\left.\log \left[\left(1-f_{u}\right) / f_{u}\right)\right]$ vs. $\log \left(D / / C_{50}\right)$. (B, $\mathbf{F}$, and $\left.\mathbf{J}\right)$ Effect of PIs on reverse transcription. qPCR was used to measure production of early reverse transcripts in primary $\mathrm{CD}^{+} \mathrm{T}$ lymphoblasts infected with pseudoviruses carrying an X4-tropic Env truncated in the CT of gp41 $\mathrm{CT}$. Pls were present at the indicated concentration during virus production. (C, G, and $\mathbf{K}$ ) Effect of PIs on all postentry steps. Flow cytometry was used to detect infection of primary CD4+ $T$ lymphoblasts by pseudoviruses carrying a VSV-G. PIs were present at the indicated concentration during virus production. ( $\mathbf{D}, \mathbf{H}$, and $\mathbf{L}$ ) Reconstruction of overall dose-response curve of Pls by combining the dose-response curves at entry and postentry steps. A 2-step form of Equation 3 was used to combine best fit dose-response curves for PI effects on entry (blue line) and all postentry steps (red line). The resulting curves (dotted black lines) were compared with experimental results for the inhibition of infectivity by Pls (black circles).

\section{Figure 2}

log-log dose-response curves illustrating the effects of the PIs ATV, DRV, and LPV on budding and entry. (A, C, and E) Budding was assessed by quantifying virus particles in the supernatants of cultures of 293T cells transfected with a proviral construct. (B, D, and F) Entry was measured by FRET using BLAM-vpr-loaded pseudoviruses with WT HIV-1 Env (filled circles), HIV-1 Env with a truncated CT (open circles), or VSV-G (triangles). Drug concentrations were normalized by previously measured $I C_{50}$ values for inhibition of infectivity by each drug (13.6 nM, 23.6 nM, and 35.8 nM for ATV, DRV, and LPV, respectively, ref. 2).

cells were washed and treated with the broad specificity endonuclease Benzonase to remove residual plasmid DNA. After 3 washes in cold PBS, the cells were lysed, and DNA was isolated for quantitation of early, intermediate, and late reverse transcripts by qPCR (38). The primers used to detect early RT products also detect intermediate and late products, and thus measurement of early products can be used to determine the overall production of proviral DNA in recently infected cells. The ability to wash cells thoroughly in cold PBS in addition to the nuclease treatment allowed us to remove most, if not all, of the plasmid DNA contamination. This is in contrast to the above situation, in which virus particles are not easily separable from contaminating plasmid DNA. In control experiments, we showed that the detection of reverse transcripts was largely blocked by addition of the fusion inhibitor enfuvirtide at the time of infection. In addition, no RT products were detected when a pseudovirus with an inactivating

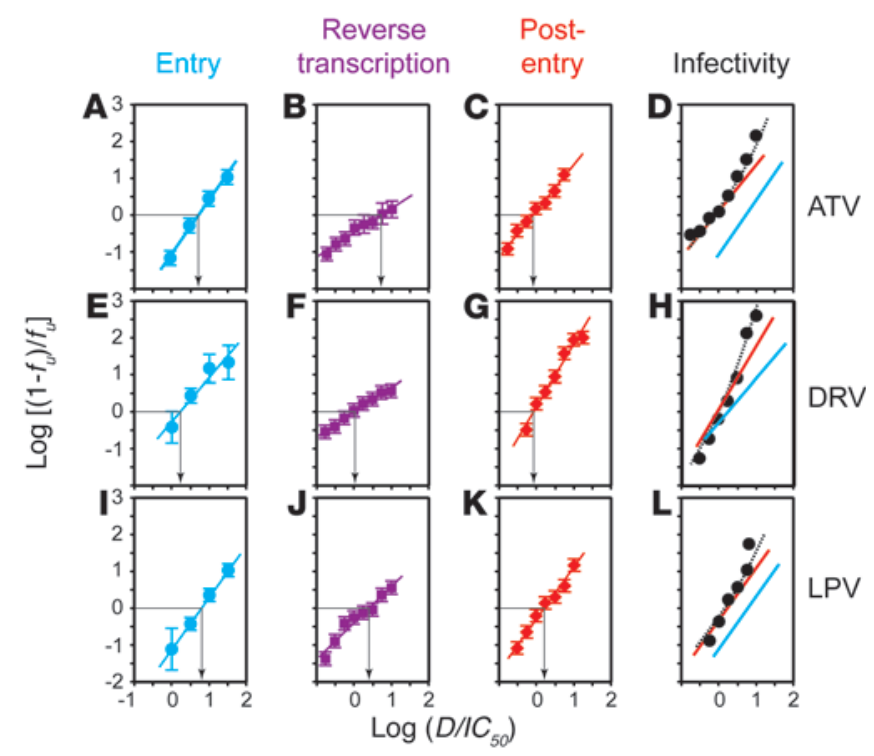




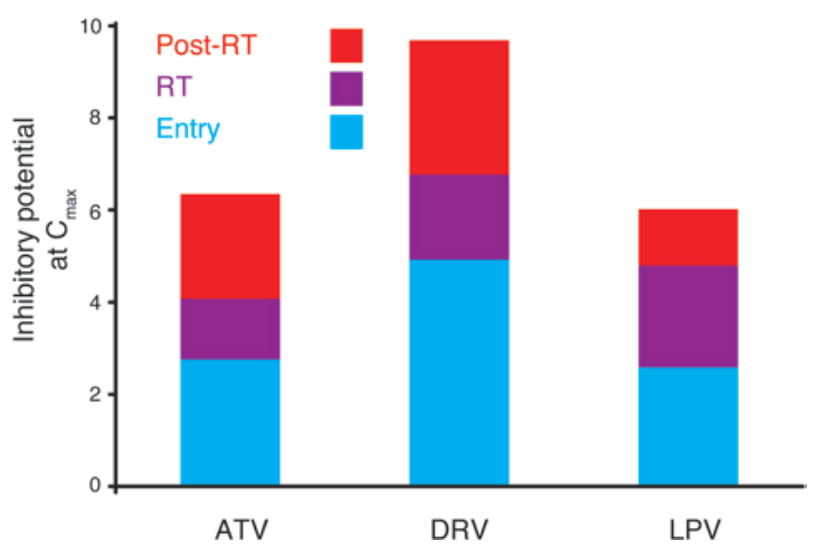

mutation in RT was used (Supplemental Figure 5). These results demonstrate that the assay detects newly synthesized viral DNA in recently infected cells.

Using this approach, we determined whether commonly used PIs affect reverse transcription. Figure 3, B, F, and J, shows doseresponse curves for PI-mediated inhibition of reverse transcription. To varying degrees, all PIs tested inhibited the appearance of reverse transcripts in a system where the entry block is largely bypassed.

Following reverse transcription, the nascent viral DNA is integrated into cellular DNA, a process catalyzed by the viral enzyme IN. IN is produced by protease-mediated cleavage of the Pr160 Gag-Pol precursor protein. Therefore, PIs can in principle also inhibit integration by preventing the formation of functional IN. Due to the heterogeneous nature of HIV-1 integration sites within the human genome $(39,40)$, available assays for integration, such as Alu-PCR-based assays (41-43), lack the precision required for analyzing the dose-response curves of PIs at the integration step. Instead, we utilized VSV-G pseudotyped viruses, which are inhibited only at postentry steps (Figure 2), to quantify the combined inhibition by PIs of all postentry steps, including integration (Figure 3). For all PIs tested, dose-response curves for the combined inhibition of all postentry steps were significantly steeper than the curves for inhibition of reverse transcription $(P=0.0086$, 0.00066 , and 0.022 for ATV, DRV, and LPV, respectively), indicating that inhibition at a post-reverse transcription step is also contributing to the overall inhibition (Figure 3). Interestingly, dose-response curves for the combined inhibition at all postentry steps also showed lower $I C_{50}$ values than the $I C_{50}$ values for the inhibition of entry. Taken together, these results demonstrate that PIs affect multiple distinct steps in the life cycle including both entry and postentry events.

Reconstructing PI dose-response curves based on inbibition at individual steps of the viral life cycle. Having separately measured inhibition by PIs at entry and post entry steps, we determined whether the overall dose-response curves of PIs could be predicted by assuming that the fraction of successful infection events is the product of the fraction of events unaffected at each of the relevant steps in the life cycle (Figure 1, B and C, Supplemental Appendix 1, and Supplemental Figure 6). This is the same assumption in the Bliss model of drug-drug interaction that is used to predict the overall inhibition achieved by 2 drugs inhibiting different step in the life cycle $(4,27)$. Using the Bliss model to combine entry and postentry inhibition by the PIs, we successfully predicted the overall doseresponse curves for all PIs tested. Results for the most commonly

\section{Figure 4}

Contribution of the inhibitory effect of PIs on each step of viral life cycle to the overall inhibitory effect at $C_{\max }$. The linear dose-response curves of PIs at entry, reverse transcription, and post-reverse transcription steps were extrapolated to predict the inhibition of each step at $C_{\max }$.

used PIs (ATV, DRV, LPV) are shown in Figure 3. The theoretical curves matched the experimentally obtained dose-response curves with $R^{2}$ values of $0.97,0.94$, and 0.97 for ATV, DRV, and LPV, respectively. The upward inflection of the overall dose-response curves reflects the fact that at higher concentrations, infection is blocked at the entry step as well as at postentry steps. Thus, the unique pharmacodynamic features of PI dose-response curves, i.e., the steepness and concave shape, can be explained by the fact that viruses generated in the presence of the PIs are inhibited at multiple steps in the life cycle.

We also examined the dose-response curve for PI-mediated inhibition of the generation of the Gag subunit p24, which requires multiple cleavages of the Gag precursor protein during virus maturation (Supplemental Figure 7). The dose-response curve for inhibition of this process is steep $(m=2.0)$, likely reflecting the participation of multiple copies of HIV-1 protease in the maturation of each virion (6). There is no upward inflection because this curve reflects a biochemical process and not the combined effects of inhibition at multiple independent steps in the life cycle.

At clinical concentrations, the entry inhibition by PIs is a major component of their overall inhibitory potential. Since the median effect plots of the dose-response curves of PIs at individual steps of the life cycle are linear (Figure 3), we extrapolated these plots to estimate the degree of inhibition of each individual step of the life cycle at the peak plasma concentration of each drug $\left(C_{\max }\right)$. Inhibition at post-reverse transcription steps was determined by assuming that the postentry inhibition results from independent inhibition at reverse transcription and post-reverse transcription steps. Since the inhibition at reverse transcription and the combined inhibition at all postentry steps were measured directly (Figure 3), we used the Bliss independence model to obtain the inhibition at post-RT steps. Figure 4 shows the fraction of the overall inhibition that occurs at each step in the life cycle for commonly used PIs at their peak plasma concentration, $C_{\max }$. DRV causes 9.2 logs of inhibition of new infection events at $C_{\max }, 4.2 \mathrm{logs}$ of which are due to inhibition of entry and $2.0 \mathrm{logs}$ of which are due to inhibition of reverse transcription. The remainder reflects inhibition of post-reverse transcription steps. A similar breakdown was observed for the other commonly used PIs. For each PI tested, inhibition at the entry step was most prominent, with inhibition at the reverse transcription and post-reverse transcription steps accounting for smaller and variable fractions of the total inhibition. These results demonstrate the importance of the entry inhibition in the overall effect of PIs. 


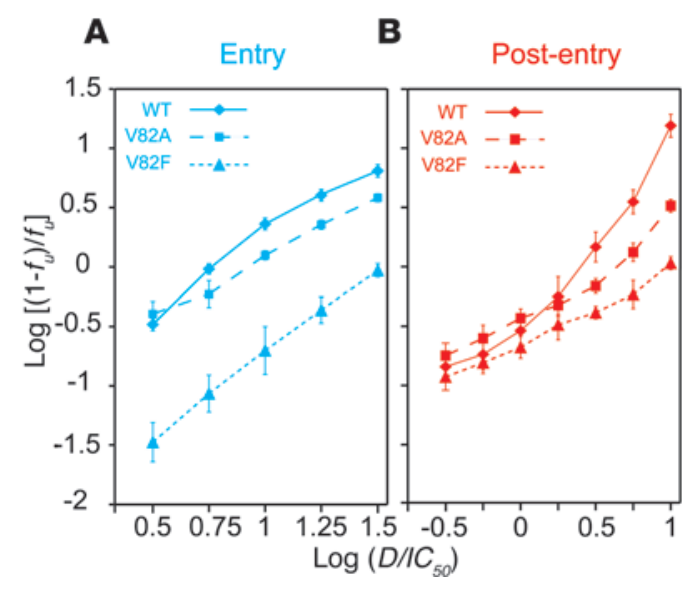

Because the effects of PIs on entry are evident at higher drug concentrations, the relative contributions of inhibition at entry and postentry steps to the overall inhibitory effect of PIs vary with drug concentration. It is expected that entry inhibition will contribute to antiviral effect in vivo throughout the interval between doses, as drug concentrations are not expected to decline to levels lacking this effect. However, drug concentrations may decrease to levels that have a decreased antientry effect during prolonged periods (2-4 days) of poor adherence. Therefore, with poor adherence, antientry effects may make a smaller relative contribution to the overall inhibition mediated by PIs.

Drug-resistance mutations in the protease gene alter both entry and postentry dose-response curves. Due to the significant contribution of entry inhibition to the overall inhibition produced by PIs (Figure 4), we hypothesized that drug-resistance mutations would alter dose-response curves for both the entry and postentry events. To test this hypothesis, we introduced 2 LPV resistance mutations, V82A and $\mathrm{V} 82 \mathrm{~F}(15)$, into the NL4-3 $\triangle$ Env provirus by site-directed mutagenesis to create NL4-3 EnvV82A and NL4-3 $\Delta$ EnvV82F, respectively. $293 \mathrm{~T}$ cells were cotransfected with NL4-3 $\mathrm{EnvV82A}$ or NL4-3 $\Delta$ EnvV82F, along with either a VSV-G expression vector or an HIV-1 Env expression vector and the BLAM-Vpr construct. HIV-1 Env-pseudotyped particles incorporating the BLAM-Vpr fusion protein were used to study the effect of protease resistance mutations on the inhibition of HIV-1 entry by LPV (37), and the VSV-G pseudotyped particles were used to study the inhibition at postentry steps using a single-round infectivity assay with GFP expression as the readout (44). The results are shown in Figure 5, $\mathrm{A}$ and $\mathrm{B}$. These mutations produced resistance by altering both entry and postentry dose-response curves. However, as shown in the next section, one of the 2 commercial phenotypic assays now in widespread clinical use is not capable of accurately measuring the effect of PIs on entry because an HIV-1 Env is not used.

Importance of the HIV-1 Env protein in the measurement of resistance to PIs. Genotypic and phenotypic assays for drug resistance play an important role in the management of HIV-1 infection. In the most commonly used phenotypic assay (45), a $1.5-\mathrm{kb}$ region of the pol gene spanning the $\mathrm{p} 7-\mathrm{p} 1-\mathrm{p} 6$ protease cleavage sites in Gag, protease, and a portion of the RT coding region is cloned into an indicator HIV-1 vector expressing luciferase. Pseudoviruses are made in $293 \mathrm{~T}$ cells by cotransfecting this vector with an expression vector encoding an amphotropic murine leukemia virus envelope protein (MLV-E). The resulting pseudoviruses are then used to

\section{Figure 5}

Effect of PI-resistance mutations in the protease gene on inhibition of entry and postentry steps of viral life cycle. (A) Effect of LPV-resistance mutations in the protease gene on HIV-1 entry. 293T cells were cotransfected with an NL4-3 $\Delta$ Env vector expressing either WT protease or one with the protease mutations $\mathrm{V} 82 \mathrm{~A}$ or $\mathrm{V} 82 \mathrm{~F}$, a vector expressing an X4-tropic HIV-1 envelope, and Blam-Vpr. Viruses were produced in the presence of increasing concentrations of LPV, and a highly sensitive FRET-based entry assay was then used to quantitate the amount of entry into primary $\mathrm{CD}^{+}{ }^{+} \mathrm{T}$ cells. (B) Effect of PI-resistance mutations in the protease gene on postentry events. 293T cells were cotransfected with an NL4-3 $\Delta$ Env vector expressing either WT protease or one with the protease mutations V82A or V82F, and a vector expressing VSV-G. The transfected cells were then plated in 96-well plates, and LPV was added. Two days after the transfection, the viral supernatant was used to infect CD4+ lymphoblasts. Three days after infection, GFP-expressing cells were quantified using flow cytometry.

infect fresh $293 \mathrm{~T}$ cells. Dose-response curves for the inhibition of infection by pseudoviruses carrying patient-derived pol sequences are compared with curves for a WT reference strain. A shift in the $I C_{50}$ greater than an empirically determined value is reported as evidence of phenotypic resistance (45).

The transmembrane subunit of the MLV-E is a $15-\mathrm{kDa}$ protein ( $\mathrm{p} 15$ ) that is cleaved in the cytoplasmic domain by the MLV protease into a $12-\mathrm{kDa}$ protein (p12) and a 16-residue peptide (p2). This cleavage activates the fusion potential of MLV-E. When Env-defective HIV-1 proviruses are pseudotyped with MLV-E, the HIV-1 protease cleaves $\mathrm{p} 15$ and renders the envelope fusogenic $(12,46)$. We hypothesized that PIs might inhibit the entry of MLV-E-pseudotyped particles by preventing this essential cleavage. Such an inhibitory effect on a clinically irrelevant substrate, the MLV-E, could artificially alter the dose-response curves of the PIs and compromise the analysis of resistance.

To evaluate this hypothesis, we tested the effect of HIV-1 protease enzyme function on entry of HIV-1 pseudoviruses expressing either the WT MLV-E (p15) or a mutant form of MLV-E (p12) with a stop codon at the protease cleavage site. To generate these pseudoviruses, $\mathrm{p} 15$ or $\mathrm{p} 12$ expression vectors were cotransfected into $293 \mathrm{~T}$ cells along with the BLAM- $\mathrm{VPr}$ vector and the NL4-3 $\Delta$ Env proviral construct encoding either WT protease or protease with an inactivating active site mutation (D25N). After normalization based on viral RNA copy number, the resulting pseudoviruses were used to infect activated primary $\mathrm{CD}^{+}$cells, and entry was measured using the FRET-based fusion assay. Figure 6A shows that functional HIV-1 protease is essential for entry of pseudoviruses with MLV-E since proteasedefective pseudoviruses are unable to enter. This entry block is alleviated by truncating the MLV-E CT at the site of protease cleavage (Figure 6A), consistent with previous studies (46).

To determine whether HIV-1 PIs inhibit the entry of MLV-E pseudoviruses, we transfected 293T cells with expression vectors for MLV-E and BLAM-Vpr, and with the NL4-3 $\Delta$ Env proviral construct in the presence of the PI DRV. After 48 hours, supernatants containing newly generated virus particles were used to infect activated primary $\mathrm{CD}^{+} \mathrm{T}$ cells. Figure $6 \mathrm{~B}$ shows that DRV inhibits the entry of pseudoviruses carrying WT MLV-E (p15). In contrast, entry mediated by the truncated form of MLV-E (p12) was only weakly inhibited by DRV. Clinical assays to detect protease resistance use pseudoviruses containing MLV-E. To investigate the extent to which the use of MLV-E affects the overall dose- 
A

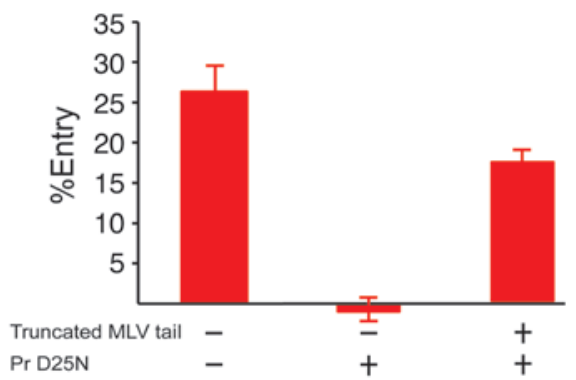

Infectivity

MLV-E

$\mathrm{CD}^{+} \mathrm{T}$ cells
Infectivity

MLV-E 293/CD4*T cells
Infectivity

MLV-E/HIV Env 293/CD4* T cells

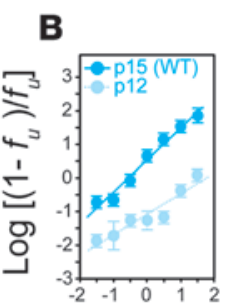

C

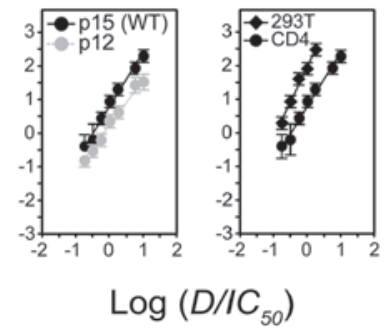

E

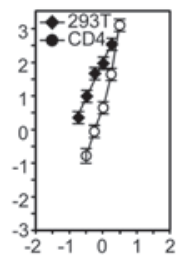

\section{Figure 6}

Importance of the entry effect on the analysis of resistance to PIs. (A) Dependence of pseudoviruses with MLV-E on HIV-1 protease for entry. HIV-1 pseudoviruses with WT or mutant (D25N) protease and the indicated forms of MLV-E were used to infect CD4+ lymphoblasts, and entry was measured by FRET. (B) Effect of PI treatment of virus-producing cells on the entry of pseudoviruses with MLV-E. Pseudoviruses with the indicated forms of MLV-E and WT protease were generated in the presence of increasing concentrations of DRV and tested for entry into primary CD4+ $\mathrm{T}$ lymphoblasts using FRET. (C) DRV-mediated inhibition of infection of CD4+ $\mathrm{T}$ lymphoblasts by HIV-1 pseudoviruses with WT (p15) or truncated (p12) MLV-E. Infection was assessed by GFP expression in target cells. (D) The effect of target cell type on PI dose-response curves. HIV-1 pseudoviruses with WT MLV-E generated in the presence of increasing concentrations of DRV were used to infect $293 \mathrm{~T}$ cells or primary CD4+ T lymphoblasts. Infection was assessed by GFP expression in target cells. (E) Comparison of DRV dose-response curves in experimental systems representing the clinical assay for resistance (MLV-E/293T) or in vivo infection (HIV-1 Env/ CD4). HIV-1 pseudoviruses with MLV-E or HIV-1 Env were generated in the presence of increasing concentrations of DRV and used to infect 293T cells or primary CD4+ T lymphoblasts. Infection was assessed by GFP expression in target cells. response curves of PIs, we examined DRV-mediated inhibition of the infection of $293 \mathrm{~T}$ cells or primary $\mathrm{CD}^{+} \mathrm{T}$ cells by recombinant HIV-1 viruses carrying MLV-E or HIV-1 Env. The readout was the percentage of GFP-positive cells at 2 days after infection. As shown in Figure 6C, dose-response curves for inhibition of infection of CD4 ${ }^{+} \mathrm{T}$ cells by MLV-E pseudoviruses were steeper than the curves for inhibition of entry (Figure 6B). This likely reflects the fact that infectivity assays capture both entry and postentry events. Truncation of the CT had a smaller effect on the dose-response curve for infectivity than on the dose-response curve for entry (compare Figure 6, B and C). Again this is expected because the infectivity assay captures effects of the inhibition of protease function on multiple steps including entry, reverse transcription, and post- reverse transcription steps. Cell-type differences are also apparent in the dose-response curves (Figure 6D), with inhibition of infection of 293 T cells by MLV-E pseudoviruses occurring at lower DRV concentrations than those required for inhibition of infection of $\mathrm{CD}^{+} \mathrm{T}$ cells. Finally, we compared the dose-response curves for inhibition of MLV-E pseudovirus infection of 293T cells and for inhibition of HIV-1 Env pseudovirus infection of primary $\mathrm{CD}^{+} \mathrm{T}$ cells (Figure $6 \mathrm{E}$ ). The former represents the commercial resistance assay, while the latter more closely reflects HIV-1 infection in vivo. The dose-response curve for MLV-E pseudovirus infection of $293 \mathrm{~T}$ cells is shifted to the left by approximately $0.5 \mathrm{logs}$ and does not show the upward inflection evident in dose-response curves for HIV-1 Env pseudovirus infection of primary $\mathrm{CD}^{+} \mathrm{T}$ cells. This may reflect the fact that the effect of protease on entry is different in the 2 cases. For MLV-E, the inhibited enzyme acts directly on the envelope protein. For HIV-1 Env, the protease acts on the Gag polyprotein, which interacts with the CT of Env. The net effect is that the shapes of the dose-response curves are different. These differences could influence the interpretation of clinical assays for drug resistance. At DRV concentrations around the $I C_{50}(23.6 \mathrm{nM})$, the difference in inhibition is over 10 -fold and is highly significant $(P<0.015)$.
HIV-1 env mutations can confer PI resistance even in the context of WT gag and pol genes. PIs achieve their overall inhibition of viral replication by the combined effects of inhibition at multiple steps in the life cycle (Figure 3). Studies of Gag-Env interactions by others $(17,18)$ have shown that interactions between the gp41 CT and the MA component of Pr55 ${ }^{\mathrm{Gag}}$ have an inhibitory effect on fusion that is relieved by protease-mediated cleavage of $\operatorname{Pr} 55^{\mathrm{Gag}}$. We have shown (Figure 2) that treatment of virus-producing cells with PIs at clinical concentrations also inhibits viral fusion and that this inhibition contributes significantly to the overall inhibition produced by PIs (Figure 4). A single-point mutation that causes premature termination of gp41 largely abolishes the inhibition of entry by PIs (Figure 2). In addition, resistance mutations in the protease gene alter both entry and postentry dose-response curves (Figure 5). Therefore, we hypothesized that mutations in the env gene that alter the interaction with MA might arise in vivo. Such mutations could in principle confer some degree of PI resistance even in the context of WT gag and pol genes and provide a selective advantage to the mutant viruses. Importantly, such viruses would be identified as drug susceptible in most current clinical assays for resistance, since the env gene is not included in genotypic analyses and MLV-E is used in one of the common phenotypic assays.

To determine whether mutations in env can confer PI resistance, we examined clinical isolates with high-level resistance to PIs. Koh et al. (47) isolated HIV-1 variants from a patient failing PI-containing regimens. These viruses contained 9 to 14 protease mutations associated with PI resistance. The viruses were then grown in the presence of DRV for 51 passages and additional mutations in the protease gene accumulated (47). We cloned the full-length env gene from passages 1 and 51 (E-1 and E-51). Pseudoviruses were made in $293 \mathrm{~T}$ cells, in the presence of PIs, by cotransfecting the cells with the NL4-3 $\Delta$ Env proviral construct and expression vectors for either the E-1 or E-51 Envs. Except for the env gene, the HIV-1 genes in these constructs were WT, including the pol gene with the protease coding region and the gag gene. Figure 7 , 


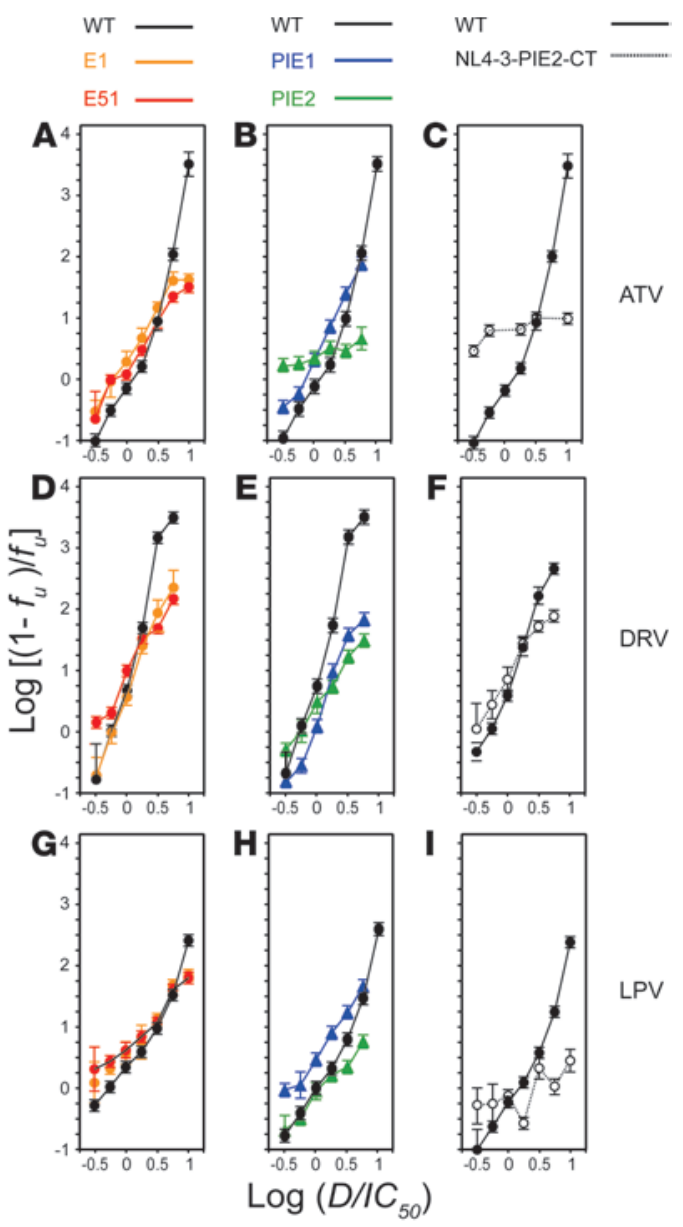

A, D, and G, shows that even in the context of WT gag and pol, these mutant Envs affect the dose-response curves for PIs, flattening the curves and producing a substantial degree of resistance at PI concentrations 10 -fold above the $I C_{50}$ for $\mathrm{WT}$ virus. Interestingly, the flattening of dose-response curves is also characteristic of single-resistance mutations in the protease gene (48). This effect was prominent for the PI DRV, but was also observed for the other commonly used PIs, ATV, and LPV. This resistance was more apparent at higher PI concentrations, consistent with the observation that the effect of PIs on entry requires higher drug concentrations than the effects on subsequent steps (Figure 3). At $132 \mathrm{nM}\left(2 \operatorname{logs}\right.$ below $C_{\max }$ at a $\log D / I C_{50}$ value of 0.75 on the $x$ axis), the fraction of infection events blocked by DRV was 10 -fold more when WT Env was used to generate pseudoviruses compared with pseudoviruses with either the E-1 or E-51 envelopes.

Next, we studied patients on PI-based regimens who had detectable viremia. None of these patients had any major PI-resistance mutations as reported by standard clinical genotypic assays (Supplemental Table 1). Full-length env genes were cloned from the plasma or PBMCs of these patients as indicated in Supplemental Table 1. Pseudoviruses were made in $293 \mathrm{~T}$ cells, in the presence of PIs, by cotransfecting the cells with the NL4-3 $\Delta$ Env proviral construct and expression vectors for patient-derived Envs. Except for the env gene, the HIV-1 genes in these constructs were WT, including the pol gene with the protease coding region and the gag gene. A total of 18 distinct functional env clones from 6 different patients

\section{Figure 7}

Effect of env mutations on PI resistance. (A, D, and G) Mutations in Env can confer PI resistance. Pseudoviruses were generated with WT gag and pol genes and env genes E1 or E51 from a patient with high level PI resistance. Pseudoviruses were produced in the presence of various concentrations of the indicated PIs and used to infect CD4+ lymphoblasts. Three days later, the infection was quantified as the percentage of cells expressing GFP. Control WT infections were done with pseudoviruses carrying NL4-3 env. (B, E, and $\mathbf{H}$ ) Envs from patients who failed PI-containing regimens without evidence of major PI mutations confer PI resistance. Pseudoviruses were generated with WT gag and pol genes and env genes cloned from patients PIE1 and PIE2 and analyzed as described above. (C, F, and I) Effect of mutations in the gp41 CT on PI sensitivity. Pseudoviruses generated with WT gag and pol and a chimeric NL4-3 env with the CT from PIE2 (NL4-3-PIE2-CT) were analyzed as above. Dose-response curves for ATV, DRV, and LPV are shown. Drug concentrations are normalized to the $I C_{50}$ values for infectivity measured in (2) and are $13.6 \mathrm{nM}, 23.6 \mathrm{nM}$, and $35.8 \mathrm{nM}$ for ATV, DRV, and LPV, respectively. were studied. Figure 7, B, E, and H, shows that env genes cloned from 2 of these patients (PIE1 and PIE2) conferred significant PI resistance even in the context of WT gag and pol genes. As in the example above, this resistance was apparent at $132 \mathrm{nM} \mathrm{DRV}$, which is well below the $C_{\max }$ value of $15 \mu \mathrm{M}$ (2).

Several different parameters were evaluated to assess the level of PI resistance conferred by patient-derived env sequences (Table 1 and Supplemental Table 2). As indicated in Equation 1, antiviral activity is influenced by both the $I C_{50}$ and the dose-response curve slope $(m)$. Changes in either parameter can cause resistance to antiretroviral drugs, with decreases in $m$ being particularly important for PI resistance (48). Instantaneous inhibitory potential (IIP) is a parameter that takes into account both $I C_{50}$ and $m$. IIP is the number of logs by which a particular concentration of drug reduces single-round infection (2). Resistance is manifest as a decrease in IIP. Table 1 shows that 9 of 18 patient-derived env clones conferred significant DRV resistance as assessed by a statistically significant fractional decrease in IIP at $C_{\max }$. Similar results were obtained for ATV and LPV (Supplemental Table 2). Resistance mutations can decrease the replication capacity of the mutant virus in the absence of drug. Thus the actual selective advantage that a mutation confers in the presence of drugs can be calculated by considering both the change in replication capacity and the degree of resistance (48). Selective advantage is defined as the ratio of the replication rate of mutant virus to the replication rate of the WT virus under a given set of conditions. Table 1 also lists replication capacity and selec- 
Table 1

Summary of the dose-response curve parameters for patient-derived Env clones

\begin{tabular}{|c|c|c|c|c|c|c|c|c|c|}
\hline Patient & Clone & $\mathrm{X} 4 / \mathrm{R5}^{\mathrm{A}}$ & Drug & $I C_{50}(\mathrm{nM})^{\mathrm{B}}$ & Slope ${ }^{B}$ & $I I P_{C \max }{ }^{\mathrm{C}}$ & $\begin{array}{l}\text { Fractional change } \\
\text { in } I I P_{C \max } \mathrm{D}\end{array}$ & $\begin{array}{l}\text { Replication } \\
\text { capacity }\end{array}$ & $\begin{array}{c}\text { Selective } \\
\text { advantage }^{\mathrm{F}}\end{array}$ \\
\hline & HXB & $X 4$ & DRV & $12.23 \pm 0.68$ & $3.05 \pm 0.32$ & $9.60 \pm 0.75^{\mathrm{G}}$ & $N A$ & 1.00 & 1.00 \\
\hline & SF162 & RV & DRV & $13.60 \pm 0.41$ & $3.29 \pm 0.20$ & $9.21 \pm 0.55$ & $0.04 \pm 0.00$ & $0.07 \pm 0.05$ & $0.18 \pm 0.03$ \\
\hline $\mathrm{CP}$ & $98 \mathrm{rd12}$ & R5 & DRV & $20.88 \pm 0.47$ & $3.43 \pm 0.17$ & $9.59 \pm 0.49$ & $0.00 \pm 0.00$ & $0.48 \pm 0.06$ & $0.49 \pm 1.01$ \\
\hline \multirow[t]{3}{*}{ HAART } & 2522 & R5 & DRV & $18.10 \pm 0.78$ & $3.07 \pm 0.29$ & $8.59 \pm 0.81$ & $0.11 \pm 0.01$ & $0.29 \pm 0.03$ & $2.97 \pm 7.53$ \\
\hline & E1 & $X 4$ & DRV & $13.21 \pm 0.26$ & $2.47 \pm 0.09$ & $6.90 \pm 0.24$ & $0.28 \pm 0.02$ & $0.05 \pm 0.01$ & $23.35 \pm 46.22$ \\
\hline & $\mathrm{E} 51$ & $\mathrm{X} 4$ & DRV & $6.63 \pm 0.29$ & $1.69 \pm 0.10$ & $4.72 \pm 0.28$ & $0.51 \pm 0.05$ & $0.06 \pm 0.00$ & $4269 \pm 7862$ \\
\hline PIE1 & PIE1 & R5 & DRV & $12.47 \pm 0.41$ & $1.80 \pm 0.11$ & $5.04 \pm 0.32$ & $0.48 \pm 0.05$ & $0.10 \pm 0.01$ & $3668 \pm 6879$ \\
\hline PIE2 & PIE2 & R5 & DRV & $20.75 \pm 0.26$ & $1.91 \pm 0.19$ & $5.35 \pm 0.24$ & $0.44 \pm 0.04$ & $0.01 \pm 0.00$ & $202.69 \pm 367$ \\
\hline PIE2 & CDS4 & R5 & DRV & $16.69 \pm 0.64$ & $1.83 \pm 0.15$ & $5.13 \pm 0.43$ & $0.47 \pm 0.05$ & $0.06 \pm 0.01$ & $1816 \pm 3616$ \\
\hline PIE3 & TBL-1 & R5 & DRV & $14.92 \pm 0.43$ & $1.59 \pm 0.11$ & $4.45 \pm 0.32$ & $0.54 \pm 0.10$ & $0.32 \pm 0.06$ & $46027 \pm 86460$ \\
\hline PIE4 & $15-9$ & R5 & DRV & $13.05 \pm 0.28$ & $2.89 \pm 0.12$ & $8.08 \pm 0.34$ & $0.16 \pm 0.01$ & $0.08 \pm 0.01$ & $2.63 \pm 4.98$ \\
\hline PIE4 & $15-13$ & R5 & DRV & $10.43 \pm 0.34$ & $2.60 \pm 0.15$ & $7.28 \pm 0.42$ & $0.24 \pm 0.02$ & $0.15 \pm 0.01$ & $31.40 \pm 62.04$ \\
\hline PIE4 & $15-17$ & R5 & DRV & $9.92 \pm 0.41$ & $2.73 \pm 0.22$ & $7.62 \pm 0.62$ & $0.21 \pm 0.02$ & $0.16 \pm 0.01$ & $15.48 \pm 34.57$ \\
\hline PIE4 & $15-20$ & R5 & DRV & $11.73 \pm 0.29$ & $2.73 \pm 0.12$ & $7.63 \pm 0.35$ & $0.21 \pm 0.02$ & $0.17 \pm 0.01$ & $15.41 \pm 29.29$ \\
\hline PIE4 & $15-22$ & R5 & DRV & $10.11 \pm 0.37$ & $2.60 \pm 0.19$ & $7.26 \pm 0.52$ & $0.24 \pm 0.03$ & $0.08 \pm 0.00$ & $16.69 \pm 35.02$ \\
\hline PIE5 & $16-2$ & $\mathrm{X} 4$ & DRV & $13.11 \pm 0.67$ & $2.60 \pm 0.26$ & $7.27 \pm 0.73$ & $0.24 \pm 0.03$ & $0.08 \pm 0.01$ & $16.04 \pm 38.52$ \\
\hline PIE5 & $16-4$ & $X 4$ & DRV & $10.40 \pm 0.27$ & $2.52 \pm 0.13$ & $7.05 \pm 0.36$ & $0.27 \pm 0.02$ & $0.03 \pm 0.00$ & $11.26 \pm 21.56$ \\
\hline PIE6 & $18-4$ & R5 & DRV & $20.43 \pm 0.80$ & $2.86 \pm 0.25$ & $8.01 \pm 0.69$ & $0.17 \pm 0.02$ & $0.34 \pm 0.02$ & $13.18 \pm 31.00$ \\
\hline PIE6 & $18-5$ & R5 & DRV & $18.88 \pm 0.64$ & $2.43 \pm 0.19$ & $6.80 \pm 0.52$ & $0.29 \pm 0.03$ & $0.19 \pm 0.02$ & $121.56 \pm 256$ \\
\hline PIE6 & $18-8$ & R5 & DRV & $5.78 \pm 1.13$ & $1.75 \pm 0.45$ & $4.89 \pm 1.27$ & $0.49 \pm 0.13$ & $0.23 \pm 0.03$ & $11985 \pm 4069$ \\
\hline PIE6 & $18-9$ & R5 & DRV & $11.73 \pm 0.29$ & $2.73 \pm 0.12$ & $7.63 \pm 0.35$ & $0.21 \pm 0.02$ & $0.30 \pm 0.03$ & $28.07 \pm 53.41$ \\
\hline PIE6 & $18-10$ & R5 & DRV & $10.51 \pm 0.36$ & $2.03 \pm 0.12$ & $5.68 \pm 0.35$ & $0.41 \pm 0.04$ & $0.12 \pm 0.01$ & $971.8 \pm 1847$ \\
\hline PIE6 & $18-14$ & R5 & DRV & $14.69 \pm 0.52$ & $2.25 \pm 0.16$ & $6.30 \pm 0.45$ & $0.34 \pm 0.04$ & $0.17 \pm 0.02$ & $343.89 \pm 692$ \\
\hline PIE6 & $18-16$ & R5 & DRV & $16.39 \pm 0.98$ & $1.60 \pm 0.28$ & $4.46 \pm 0.78$ & $0.54 \pm 0.19$ & $0.06 \pm 0.00$ & $8225 \pm 20400$ \\
\hline
\end{tabular}

ACoreceptor usage predicted by the Geno2Pheno HIV coreceptor usage prediction algorithm (68). ${ }^{B}$ Slope and $/ C_{50}$ were calculated by fitting a linear regression model to the plot of $\log \left(\left(1-f_{u}\right) / f_{u}\right)$ vs. $\log \left(D / / C_{50}\right)$ (Equation 2). See Supplemental Appendix 2 for details. Higher slope values are obtained in analysis that considers the upward inflection in the median effect plots (see ref. 4). ${ }^{C} / I P_{C \max }$ is the number of logs of inhibition of a single round of infection at $C_{\max }$ and was calculated using Supplemental Equation 7. DFractional change in $I I P_{C \max }\left(\Delta I / P_{C \max }\right)$ for a given Env clone is calculated using Supplemental Equation 9. A positive value indicates a drug-resistant clone, whereas a negative value indicates a clone that is more susceptible than WT. The $95 \% \mathrm{Cl}$ for $\Delta / I P_{C m a x}$ is calculated as $\left[\mu-1.96^{\prime} \sigma, \mu+1.96^{\prime} \sigma\right]$, where $\mu$ is the mean and $\sigma$ is the SEM for $\Delta I I P_{C \max }$. Clones with a $95 \% \mathrm{Cl}$ for $\Delta / I P_{C \max }$ that did not include 0 for all 3 drugs tested appear in bold. EReplication capacity is the relative ability of a mutant virus to complete a single round of infection compared with the WT in the absence of a drug. It is calculated according to Supplemental Equation 11. FSelective advantage is calculated using Supplemental Equation 12. GIIP $P_{C \max }$ for the WT HXB2 was calculated by combining the 2 dose-response curves for entry and postentry steps, as described in Equations 3 and 4 .

tive advantage for the patient-derived env clones. Some of the clones show a high selective advantage over WT virus at high drug concentrations. Only $50 \%$ of the env clones analyzed conferred significant resistance, and no resistant clones were identified in samples from one patient (PIE4). Thus, Env sequences may contribute to PI failure only in a subset of patients. Pseudoviruses generated with env genes cloned from a patient on a fully suppressive HAART regimen and a treatment-naive chronic progressor did not confer any resistance to DRV (Table 1, Supplemental Table 2, and Supplemental Figure 8).

Since the gp 41 CT is required for the inhibition of entry by PIs, we hypothesized that drug-resistance mutations in the env gene could be localized to this region in at least some cases. We created constructs expressing chimeric Env proteins with the CT of gp41 from the PI-resistant viruses E-1, E-51, PIE1, or PIE2, and the remainder of gp41 and all of gp120 from a WT, X4-tropic env. Corresponding pseudoviruses (NL4-3-E1-CT, NL4-3-E51-CT, NL4-3-PIE1-CT, and NL4-3-PIE2-CT) were made by cotransfecting HEK293T cells with NL4-3 $\Delta$ Env and constructs expressing the above chimeric Envs. Figure 7, C, F, and I, shows that viruses pseudotyped with the NL4-3-PIE2-CT envelope are resistant to PIs even when the gag and pol genes and most of the env gene, with the exception of gp41 CT, are WT. Pseudoviruses made with the other chimeric envelopes demonstrated dose-response curves that were not significantly different than the WT. Therefore, in some cases mutations in the gp41 CT are sufficient to confer PI resistance. Viruses harboring only these mutations would be erroneously identified as drug-susceptible clinical assays due to the exclusion of HIV-1 env region and the use of MLV-E in generating pseudoviruses. Taken together, these results demonstrate that an accurate assessment of PI resistance may require analysis of the env gene.

\section{Discussion}

Modern HAART regimens typically consist of 3 drugs. Each drug inhibits a subset of infection events on its own; however, in combination therapy, multiple log inhibition is achieved. Very high levels of inhibition can be achieved with combinations that include drugs targeting different steps in the life cycle (4). In this situation, drugs acting at downstream steps in the life cycle block viruses that were not inhibited by drugs acting at the previous steps. A full replication cycle requires a virus to clear multiple blocks from drugs acting at different steps. The low probability with which this occurs contributes to the success of HAART (4).

Among anti-HIV-1 drugs, PIs stand out. We and others have shown that in vitro, PIs exhibit superior antiviral activity $(4,49-51)$. In addition, their dose-response curves are steep. Median effect plots of the dose-response relationship for PIs are nonlin- 
ear and inflect upward as the concentration of the drug increases, resulting in extraordinarily high levels of inhibition at concentrations only slightly above the $I C_{50}$. These unique pharmacodynamic features make PIs candidate drugs for monotherapy. In fact, PIs are the only class of antiretroviral drugs for which clinical trials have demonstrated that monotherapy is not inferior to triple-drug therapy at maintaining suppression of $\operatorname{HIV}-1$ replication $(5,52)$.

In this study, we show that in effect, PIs act like multiple drugs in one. Using what we believe to be a novel, highly sensitive budding assay, we proved that immature particles can be released efficiently from cells treated with PIs. However, these immature virions produced in the presence of PIs are incapable of efficiently completing entry, reverse transcription, and post-reverse transcription steps. A sensitive FRET-based virus-cell entry assay (37) allowed us to measure the inhibition of entry by PIs. Using pseudoviruses with a truncated gp $41 \mathrm{CT}$ allowed us to bypass the entry block and thereby isolate and measure the dose-response curves for PIs at the reverse transcription step. In addition, measuring GFP expression following infection with VSV-G pseudoviruses, which are not inhibited at entry, allowed us to measure postentry inhibition. Subtracting out the inhibition of reverse transcription from postentry inhibition allowed us to mathematically obtain the post-reverse transcription inhibition by PIs. Finally, by combining the dose-response curves of PIs at the entry and postentry steps, we were able to reconstruct the overall dose-response curves for the inhibition of infectivity by PIs. In doing so, we used the Bliss independence model (27), which is often used to predict the combined effect achieved by 2 drugs that act independently. The reconstructed dose-response curves matched those obtained experimentally. Importantly, in this analysis, no additional parameters were introduced to fit the dose-response curves. The predictions were based solely on experimental data for inhibition at individual steps, with the combined effect calculated using the Bliss independence model. We conclude that PIs achieve very high antiviral activity by blocking the viral life cycle at multiple steps. Similarly high levels of inhibition can be achieved by combinations of potent inhibitors of entry, reverse transcription, and integration (4).

It is interesting that distinct $I C_{50}$ values can be observed for inhibition of distinct life cycle steps even though the inhibition reflects the action of the same drug on the same target. PIs may block entry because cleavage of the Gag precursor protein renders the virion fusion competent. PIs block postentry steps because cleavages of the Gag-Pol precursor protein release the RT and IN enzymes. Thus, although protease is the drug target in both cases, the substrate of the inhibited enzyme is different (Gag vs. Gag-Pol). Cleavage of the Gag-Pol precursor protein generates p17, p24, and 3 different enzymes, each of which is a multimer and each of which may be required in a different amount for successful completion of the life cycle (7). It is therefore not surprising that different levels of cleavage of these substrates may be required for each of the relevant steps. Hence, the dose-response curves can be different.

Our results are in general consistent with previous studies that have separately noted effects of PIs on individual steps in the life cycle. The virions budding from PI-treated cells are morphologically aberrant and show reduced infectivity $(50,51,53-55)$. This can be partially explained by lack of processing of Pr160 Gag-Pol. This polyprotein is the precursor to the viral enzymes protease, RT, and IN. In immature viral particles, the uncleaved Pr160 Gag-Pol precursor exhibits significantly less reverse transcription activity compared with the fully cleaved form (14). Similarly, when virus- producing cells were treated with a first-generation PI (A-77003), the resulting immature viruses produced 20 -fold fewer early RT products in the infected cells compared with viruses produced by untreated cells (56). In addition, several groups have shown that immature virus particles with inactive protease (17) or Gag cleavage sites mutations $(16,18)$ are inefficient in completing entry.

The clinical management of patients taking PIs is complicated by the fact that in clinical trials, the majority of virologic failures occur without evidence of genotypic or phenotypic resistance to these drugs (20-23). There are several potential explanations for this observation. One explanation is simply that the patients are nonadherent. If the nonadherence applies to all drugs in the regimen, then the patients are expected to fail with WT virus. This virus will show drug susceptibility in all types of resistance tests. Another explanation is that, as concentrations of PIs decrease with nonadherence, their inhibitory potential rapidly falls below the level required for the selection of resistance mutations. In this situation, mutations conferring resistance to other drugs in the regimen may arise (23), but the virus will demonstrate susceptibility to PIs in all forms of resistance testing. This hypothesis was recently shown to be plausible using a mathematical model that combines viral dynamics, evolutionary principles, and pharmacokinetic and pharmacodynamics properties of the drugs (57). The lack of PI resistance in this scenario is supported by the clinical observation that patients can be successfully treated with the same PI if good adherence is restored. For example, Kempf et al. observed that with improved adherence, 25 out of 27 patients who previously failed $\mathrm{LPV} / \mathrm{r}$-containing regimens achieved virologic suppression when put back on the same PI-containing therapy (58).

An additional mechanism for PI failure without apparent resistance is described in this study. It involves mutations in the env gene that significantly affect the antiviral activity of PIs. This mechanism is important because it leads to actual resistance to the PIs. In this situation, viral isolates will show PI resistance in in vitro assays, but this resistance may not be apparent in standard commercial resistance assays. Patient-specific changes in the env gene are not considered in standard clinical assays for genotypic or phenotypic resistance. In this study, we have shown that a significant fraction of the inhibition exerted by PIs is due to effects on HIV-1 entry. For DRV, $46 \%$ of the 9.2 logs of inhibition at $C_{\max }$ was due to the inhibition of entry compared with only $22 \%$ at the reverse transcription step. We hypothesized that escape from the entry inhibition by PIs could be a pathway by which viruses acquire PI resistance. To test this hypothesis, we first showed that known drug-resistance mutations in the protease gene achieve their overall resistance phenotype by altering both the entry and postentry dose-response curves. However, one of the standard phenotypic assays does not detect this change in the inhibition of entry because it utilizes pseudoviruses with MLV-E instead of HIV-1 Env. MLV-E requires cleavage of its short CT by HIV-1 protease to mediate fusion (Figure 6 and ref. 46). Thus, the dose-response curves of PIs obtained using the MLV-E-pseudotyped particles do not include the entry step and are confounded by the effect of PIs on a clinically irrelevant substrate, the MLV-E. Given the additional complexity introduced by the use of MLV-E, it is possible that the phenotypic assay may either overestimate or underestimate the actual degree of resistance. It is also important to point out that PI resistance without mutations in protease could be due in part to mutations in Gag that directly or indirectly affect the protease cleavage sites (59-65).

We also show here, for what we believe is the first time, that 
mutations in the env gene of HIV-1 can confer PI resistance even when the gag and pol genes are WT. We cloned full-length env genes from the circulating virus of patients who failed PI-containing regimens with no known major PI-associated resistance mutations as determined by standard genotypic analysis. Pseudoviruses generated with these Envs and a WT backbone, including WT Gag and Pol, demonstrated significant PI resistance. This was especially apparent at higher drug concentrations where the effect on HIV-1 entry becomes important. We localized the relevant drug-resistance mutations in one of these patients to the gp41 CT using a chimeric env gene with gp120 and most of gp41, except the CT, from the WT NL4-3 strain and the CT from the patient isolate. This chimeric Env fully recapitulated the PI-resistance phenotype. A molecular basis for this phenomenon has been provided by a recent fluorescence nanoscopy study showing that the clustering of Env spikes that is necessary for entry may require protease-mediated cleavage of the Gag precursor protein (66). Some mutations in the gp 41 CT may preclude interactions with the uncleaved Gag precursor that normally inhibit clustering and thus entry. In the case of 3 other PI-resistant Env clones, transfer of the CT to the NL4-3 background did not transfer resistance. In these instances, resistance may involve the complex dynamics of the entire Env trimer. A further caveat is that interactions between uncleaved Gag precursor and the gp41 CT might differ between a patient-derived Gag precursor and NL4-3-derived Gag precursor. Future biochemical studies of the interactions between the gp41 $\mathrm{CT}$ and Gag may provide a molecular explanation for the forms of PI resistance described here. Because of the unstructured nature and high sequence variability of the env CT, the detection of this form of resistance may require functional tests rather than simple genotypic analysis.

By experimentally isolating each relevant step of the HIV-1 life cycle and measuring the dose-response curves for PIs at each step, we have provided here a mechanistic explanation for the unique pharmacodynamics and exceptional efficacy of PIs. In addition, our analysis pointed to the previously ignored env gene as a possible site of PI-resistance mutations. We showed that env genes cloned from primary viral isolates from patients who failed PIcontaining regimens with virus classified as WT virus could confer PI resistance. This suggests that in some cases clinicians may be falsely reassured by relying on current commercial genotypic and phenotypic resistance assays, which do not take into account mutations in the env gene. Additional studies will be needed to determine how common this phenomenon is. If these env mutations turn out to be common, modifications in current clinical assays may be necessary to accurately quantify PI resistance.

Our results may also provide a blueprint for development of new therapeutics for other pathogens. For drugs such as PIs that target proteins influencing multiple functions that a pathogen needs in order to establish a productive infection, the inhibitory effects will multiply to achieve much greater overall inhibition compared with classes of drugs that inhibit a single step in the pathogen's life cycle.

\section{Methods}

Reagents and viruses. PIs were obtained through the NIH AIDS Research and Reference Reagent Program (Division of AIDS, National Institute of Allergy and Infectious Diseases [NIAID], NIH). To facilitate a comparison of PIs with different potencies, drug concentrations were normalized by $I C_{50}$ values determined in a previous study (2). The $I C_{50}$ values are given in the legend to Figure 2. Drug concentrations $(D)$ were plotted as log
$\left(D / I C_{50}\right)$. In various experiments, drugs were used at concentrations ranging from 0.01 to $100 \times I C_{50}(0.136 \mathrm{nM}-1.36 \mu \mathrm{M}$ for ATV, $0.236 \mathrm{nM}-2.36 \mu \mathrm{M}$ for DRV, and $0.358 \mathrm{nM}-3.58 \mu \mathrm{M}$ for LPV). CCF2-AM, and Benzonase are commercially available from Invitrogen and Sigma-Aldrich, respectively. $\beta$-Lactamase antibody was purchased from Abcam and anti-p24 antibody from Santa Cruz Biotechnology Inc.

Viruses selected in vitro for DRV resistance (E1 and E51) were a gift of Hiroaki Mitsuya (NIH, Bethesda, Maryland, USA).

Plasmids and vector constructs. The plasmid pMM310 expresses E. coli $\beta$-lactamase fused to the viral accessory protein Vpr. It was obtained through the NIH AIDS Research and Reference Reagent Program, Division of AIDS, NIAID, NIH: pMM310 (cat \#11444) from Michael Miller (Merck Laboratories). $\beta$-Lactamase expression vector was obtained by inserting a stop codon at the end of beta-lactamase ORF via site-directed mutagenesis (Stratagene).

pNL4-3 $\Delta$ Env expresses HIV-1 with GFP in the ORF of the env gene (2). The RT-deficient construct (D185N) and the protease-deficient construct were obtained from pNL4-3 $\triangle$ Env via site-directed mutagenesis. The LPV resistance mutations V82A and V82F were introduced into the protease gene via site-directed mutagenesis.

The plasmid pSV-A-MLV-env expresses the amphotropic murine leukemia virus env gene (MLV-E) and was obtained through the AIDS Research and Reference Reagent Program (Division of AIDS, NIAID, NIH): SV-AMLV-env was from Nathaniel Landau and Dan Littman (New York University, New York, New York, USA).

Analysis of viral budding. HEK $293 \mathrm{~T}$ cells were cotransfected with NL4-3 $\Delta$ Env GFP and a vector expressing an X4-tropic Env using the Lipofectamin 2000 reagent (Invitrogen) according to the manufacturer's protocol. Six hours after transfection, the cells were separated by trypsinization and distributed in 96-well plates, and PIs were added. Drugs were diluted in 50\% human serum (HS), and the cell medium also contained $50 \%$ HS to recapitulate the effect of protein binding in vivo. At 48 hours after transfection, cell-free supernatant was treated with 1 unit of Benzonase (Sigma-Aldrich) for 15 minutes in $37^{\circ} \mathrm{C}$ to degrade free, extravirion RNA. Viral RNA was extracted using QIAamp Viral RNA Mini Kit (QIAGEN). Oligo-dTs were used as primers for RT using the Superscrip III enzyme (Invitrogen). Real-time PCR was performed using TaqMan PreAmp Master Mix using the following primer-probe pairs: upstream: CAGATGCTGCATATAAGCAGCTG; downstream: TTTTTTTTTTTTTTTTTTTTTTTTTTGAAGC; probe: CCTGTACTGGGTCTCTCTGG. This primer-probe set specifically amplifies HIV-1 RNAs (67).

Viral fusion assay. A modified version of the previously described assay for viral entry based on FRET (37) was utilized. Briefly, HEK 293T cells were cotransfected with NL4-3 EEnv, BLAM- $V p r$, and an envelope-expressing construct depending on the experiment. Six hours after transfection, the cells were detached by trypsinization and were distributed into 96 -well plates, and PIs were added. Drugs were diluted in 50\% HS and cell medium contained $50 \% \mathrm{HS}$, as described above. After 48 hours, virus-containing supernatants were used to infect primary $\mathrm{CD} 4^{+} \mathrm{T}$ lymphoblasts via spinoculation (2 hours, $1200 \mathrm{~g}, 25^{\circ} \mathrm{C}$ ). After allowing the entry to occur in $37^{\circ} \mathrm{C}$ for 2 hours, $\mathrm{CD} 4^{+}$ $\mathrm{T}$ cells were incubated in CCF2-AM-containing medium for 1 hour at room temperature. Cells were then washed in RPMI and incubated overnight in $\mathrm{CO}_{2}$-independent medium (Invitrogen) at room temperature. Green-to-blue color change was detected by flow cytometry to quantify viral entry.

Infectivity assay. We utilized a GFP single-round infectivity assay described before. Briefly, HEK 293T cells were cotransfected with NL4-3AEnv, BLAM$V p r$, and an envelope-expressing construct depending on the experiment. An X4 Env (from NL4-3) was used for most experiments because X4-pseudotyped viruses result in higher levels of infection of $\mathrm{CD} 4^{+} \mathrm{T}$ lymphoblasts 
in the infectivity assay and therefore provide a higher dynamic range for assessing drug inhibition. However, we have also used an R5 Env (from SF162). Dose-response curves for PI-mediated inhibition of infection by pseudoviruses carrying the reference X4 and R5 Envs were superimposable (Supplemental Figure 9). In some experiments, patient-derived Envs were used. Six hours after transfection, 293T cells were detached by trypsinization and were distributed into 96-well plates, and PIs were added. Drugs were diluted in $50 \%$ HS and cell medium contained 50\% HS, as described above. After 48 hours, virus-containing supernatants were used to infect primary CD4 ${ }^{+} \mathrm{T}$ lymphoblasts via spinoculation (2 hours, $1200 \mathrm{~g}, 25^{\circ} \mathrm{C}$ ). $\mathrm{CD} 4^{+}$ $\mathrm{T}$ lymphoblasts were prepared by stimulation of PBMCs from healthy blood donors with phytohemagglutinin and IL-2 for 3 days followed by magnetic bead purification, as previously described (2). The PI effect was exclusively at the virus production step, and the presence of PIs during the actual infection of $\mathrm{CD}^{+} \mathrm{T}$ cells had no effect on infectivity (Supplemental Figure 10). Cells were incubated at $37^{\circ}$ for 3 days to allow completion of viral life cycle and expression of GFP, which was then analyzed using flow cytometry.

Envelope cloning and generation of chimeric envelopes. Full-length HIV-1 env genes from patient isolates or samples were cloned into an expression vector as described previously (68). Based on the Geno2Pheno HIV coreceptor usage prediction algorithm (69), the E1 and E51 isolates and isolates from patient PIE5 had predicted X4 tropism while the rest of the env clones had predicted R5 tropism (Table 1). Overlap extension PCR was utilized to make constructs expressing the chimeric Env proteins with the CT of gp41 from the PI-resistant viruses E-1, E-51, PIE1, or PIE2, and the remainder of gp41 and all of gp120 from a WT, X4-tropic envelope (NL4-3). No additional nucleotides were inserted in the junction site during the cloning. In some experiments, pseudoviruses carrying gp 41 with a truncated CT were used. For these experiments, site-directed mutagenesis was used to convert codon L753 to a stop codon in the NL4-3 Env expression vector.

Statistics. The $95 \% \mathrm{CI}$ for fractional change in $I I P_{C \max }\left(\Delta I I P_{C \max }\right)$ was calculated as $[\mu-1.96 \times \sigma, \mu+1.96 \times \sigma]$, where $\mu$ is the mean and $\sigma$ is the SEM for $\Delta I I P_{\text {Cmax. }}$. Clones with $95 \% \mathrm{CI}$ for $\Delta I I P_{\text {Cmax }}$ that did not include 0 for all 3 drugs tested were considered to have significant resistance.

Study approval. Primary $\mathrm{CD} 4^{+} \mathrm{T}$ cells were isolated from peripheral blood of healthy or HIV-1-infected adults. This study was approved by the Johns Hopkins Institutional Review Board, and all study participants provided informed consent.

\section{Acknowledgments}

We would like to thank Joel N. Blankson for his constructive suggestions. Also, we are very grateful to Hiroaki Mitsuya of the NIH for his generous gift of viruses selected in vitro for DRV resistance. This work was supported by NIH grant 081600 and the Howard Hughes Medical Institute.

Received for publication October 29, 2012, and accepted in revised form May 30, 2013.

Address correspondence to: Robert F. Siliciano, Edward D. Miller Research Building, Room 871, 733 N. Broadway, Johns Hopkins University School of Medicine, Baltimore, Maryland 21205, USA. Phone: 410.955.2958; Fax: 443.287.6218; E-mail: rsiliciano@jhmi.edu.
1. Thompson MA, et al. Antiretroviral treatment of adult HIV infection: 2010 recommendations of the international AIDS society-USA panel. JAMA. 2010; 304(3):321-333.

2. Shen L, et al. Dose-response curve slope sets classspecific limits on inhibitory potential of anti-HIV drugs. Nat Med. 2008;14(7):762-766.

3. Anderson J, Schiffer C, Lee SK, Swanstrom R. Viral protease inhibitors. Handb Exp Pharmacol. 2009; (189):85-110.

4. Jilek BL, et al. A quantitative basis for antiretroviral therapy for HIV-1 infection. Nat Med. 2012; 18(3):446-451.

5. Perez-Valero I, Arribas JR. Protease inhibitor monotherapy. Curr Opin Infect Dis. 2011;24(1):7-11.

6. Shen L, et al. A critical subset model provides a conceptual basis for the high antiviral activity of major HIV drugs. Sci Transl Med. 2011;3(91):91ra63.

7. Swanstrom R, Wills JW. Synthesis, assembly, and processing of viral proteins. In: Coffin JM, Hughes SH, Varmus HE, eds. Retroviruses. Cold Spring Harbor, New York, USA: Cold Spring Harbor Laboratory Press; 1997.

8. McCune JM, et al. Endoproteolytic cleavage of gp160 is required for the activation of human immunodeficiency virus. Cell. 1988;53(1):55-67.

9. Wlodawer A, Erickson JW. Structure-based inhibitors of HIV-1 protease. Annu Rev Biochem. 1993; 62:543-585

10. Gulnik S, Erickson JW, Xie D. HIV protease: Enzyme function and drug resistance. Vitam Horm. 2000; 58:213-256.

11. Ali A, et al. Molecular basis for drug resistance in HIV-1 protease. Viruses. 2010;2(11):2509-2535.

12. Henderson GJ, et al. Interplay between single resistance-associated mutations in the HIV-1 protease and viral infectivity, protease activity, and inhibitor sensitivity. Antimicrob Agents Chemother. 2012; 56(2):623-633.

13. Kawamura M, et al. Cleavage of gag precursor is required for early replication phase of HIV-1. FEBS Lett. 1997;415(2):227-230.
14. Lori F, et al. Enzymatically active forms of reverse transcriptase of the human immunodeficiency virus. AIDS Res Hum Retroviruses. 1988;4(5):393-398.

15. Muller B, et al. HIV-1 gag processing intermediates trans-dominantly interfere with HIV-1 infectivity. J Biol Chem. 2009;284(43):29692-29703.

16. Davis MR, Jiang J, Zhou J, Freed EO, Aiken C. A mutation in the human immunodeficiency virus type 1 gag protein destabilizes the interaction of the envelope protein subunits gp120 and gp41.J Virol. 2006;80(5):2405-2417.

17. Murakami T, Ablan S, Freed EO, Tanaka Y. Regulation of human immunodeficiency virus type 1 env-mediated membrane fusion by viral protease activity. J Virol. 2004;78(2):1026-1031.

18. Wyma DJ, et al. Coupling of human immunodeficiency virus type 1 fusion to virion maturation: A novel role of the gp 41 cytoplasmic tail. JVirol. 2004; 78(7):3429-3435.

19. Johnson VA, et al. 2011 update of the drug resistance mutations in HIV-1. Top Antivir Med. 2011; 19(4):156-164.

20. Gallego O, et al. Drug resistance in patients experiencing early virological failure under a triple combination including indinavir. AIDS. 2001; 15(13):1701-1706.

21. Havlir DV, et al. Drug susceptibility in HIV infection after viral rebound in patients receiving indinavir-containing regimens. JAMA. 2000; 283(2):229-234.

22. Pulido F, Arribas J, Hill A, Moecklinghoff C. No evidence for evolution of protease inhibitor resistance from standard genotyping, after 3 years of treatment with darunavir/ritonavir, with or without nucleoside analogues. AIDS Res Hum Retroviruses. 2012;28(10):1167-1169.

23. Taiwo B, et al. Efficacy of a nucleoside-sparing regimen of darunavir/ritonavir plus raltegravir in treatment-naive HIV-1-infected patients (ACTG A5262). AIDS. 2011;25(17):2113-2122.

24. Chou TC, Talalay P. Quantitative analysis of doseeffect relationships: The combined effects of mul- tiple drugs or enzyme inhibitors. Adv Enzyme Regul. 1984;22:27-55

25. Weiss JN. The hill equation revisited: Uses and misuses. FASEB J. 1997;11(11):835-841.

26. Hill $\mathrm{A}$. The possible effects of the aggregation of the molecules of haemoglobin on its dissociation curves. J Physiol. 1910;40(7):iv-vii.

27. Bliss CI. The toxicity of poisons jointly applied. Ann Appl Biol. 1939;26:585-615.

28. Balasubramaniam M, Freed EO. New insights into HIV assembly and trafficking. Physiology (Bethesda). 2011;26(4):236-251

29. Hong SS, Boulanger P. Assembly-defective point mutants of the human immunodeficiency virus type 1 gag precursor phenotypically expressed in recombinant baculovirus-infected cells. J Virol. 1993;67(5):2787-2798.

30. Gottlinger HG, Sodroski JG, Haseltine WA. Role of capsid precursor processing and myristoylation in morphogenesis and infectivity of human immunodeficiency virus type 1. Proc Natl Acad SciUS A. 1989; 86(15):5781-5785.

31. Smith AJ, Cho MI, Hammarskjold ML, Rekosh D. Human immunodeficiency virus type 1 Pr55gag and Pr160gag-pol expressed from a simian virus 40 late replacement vector are efficiently processed and assembled into viruslike particles. J Virol. 1990; 64(6):2743-2750.

32. Pajonk F, Himmelsbach J, Riess K, Sommer A, McBride WH. The human immunodeficiency virus (HIV)-1 protease inhibitor saquinavir inhibits proteasome function and causes apoptosis and radiosensitization in non-HIV-associated human cancer cells. Cancer Res. 2002;62(18):5230-5235.

33. Gupta AK, Li B, Cerniglia GJ, Ahmed MS, Hahn SM, Maity A. The HIV protease inhibitor nelfinavir downregulates akt phosphorylation by inhibiting proteasomal activity and inducing the unfolded protein response. Neoplasia. 2007;9(4):271-278.

34. Piccinini $\mathrm{M}$, et al. The human $26 \mathrm{~S}$ proteasome is a target of antiretroviral agents. AIDS. 2002; 16(5):693-700. 
35. Ott DE, Coren LV, Sowder RC 2nd, Adams J, Schubert U. Retroviruses have differing requirements for proteasome function in the budding process. JVirol. 2003;77(6):3384-3393.

36. Schubert $U$, et al. Proteasome inhibition interferes with gag polyprotein processing, release, and maturation of HIV-1 and HIV-2. Proc Natl Acad Sci U S A. 2000;97(24):13057-13062.

37. Cavrois M, De Noronha C, Greene WC. A sensitive and specific enzyme-based assay detecting HIV-1 virion fusion in primary $\mathrm{T}$ lymphocytes. Nat Biotechnol. 2002;20(11):1151-1154.

38. Zack JA, Arrigo SJ, Weitsman SR, Go AS, Haislip A, Chen IS. HIV-1 entry into quiescent primary lymphocytes: Molecular analysis reveals a labile, latent viral structure. Cell. 1990;61(2):213-222.

39. Schroder AR, Shinn P, Chen H, Berry C, Ecker JR, Bushman F. HIV-1 integration in the human genome favors active genes and local hotspots. Cell. 2002;110(4):521-529.

40. Shan L, et al. Influence of host gene transcription level and orientation on HIV-1 latency in a primary-cell model. J Virol. 2011;85(11):5384-5393.

41. Butler SL, Hansen MS, Bushman FD. A quantitative assay for HIV DNA integration in vivo. Nat Med. 2001;7(5):631-634.

42. O'Doherty U, Swiggard WJ, Jeyakumar D, McGain $\mathrm{D}$, Malim MH. A sensitive, quantitative assay for human immunodeficiency virus type 1 integration. J Virol. 2002;76(21):10942-10950.

43. Brussel A, Delelis O, Sonigo P. Alu-LTR real-time nested PCR assay for quantifying integrated HIV-1 DNA. Methods Mol Biol. 2005;304:139-154.

44. Zhang H, et al. Novel single-cell-level phenotypic assay for residual drug susceptibility and reduced replication capacity of drug-resistant human immunodeficiency virus type 1. J Virol. 2004; 78(4):1718-1729.

45. Petropoulos CJ, et al. A novel phenotypic drug susceptibility assay for human immunodeficiency virus type 1. Antimicrob Agents Chemother. 2000; 44(4):920-928

46. Kiernan RE, Freed EO. Cleavage of the murine leukemia virus transmembrane env protein by human immunodeficiency virus type 1 protease: Transdominant inhibition by matrix mutations. J Virol.
1998;72(12):9621-9627.

47. Koh Y, et al. In vitro selection of highly darunavirresistant and replication-competent HIV-1 variants by using a mixture of clinical HIV-1 isolates resistant to multiple conventional protease inhibitors. JVirol. 2010;84(22):11961-11969.

48. Sampah ME, Shen L, Jilek BL, Siliciano RF. Doseresponse curve slope is a missing dimension in the analysis of HIV-1 drug resistance. Proc Natl Acad Sci US A. 2011;108(18):7613-7618.

49. Shen L, Siliciano RF. Viral reservoirs, residual viremia, and the potential of highly active antiretroviral therapy to eradicate HIV infection. J Allergy Clin Immunol. 2008;122(1):22-28.

50 . Kempf DJ, et al. Antiviral and pharmacokinetic properties of $\mathrm{C} 2$ symmetric inhibitors of the human immunodeficiency virus type 1 protease. Antimicrob Agents Chemother. 1991;35(11):2209-2214.

51. McQuade TJ, et al. A synthetic HIV-1 protease inhibitor with antiviral activity arrests HIV-like particle maturation. Science. 1990;247(4941):454-456.

52. Bierman WF, van Agtmael MA, Nijhuis M, Danner SA, Boucher CA. HIV monotherapy with ritonavirboosted protease inhibitors: a systematic review. AIDS. 2009;23(3):279-291.

53. Ashorn P, McQuade TJ, Thaisrivongs S, Tomasselli AG, Tarpley WG, Moss B. An inhibitor of the protease blocks maturation of human and simian immunodeficiency viruses and spread of infection. Proc Natl Acad Sci U S A. 1990;87(19):7472-7476.

54. Erickson J, et al. Design, activity, and 2.8 A crystal structure of a C2 symmetric inhibitor complexed to HIV-1 protease. Science. 1990;249(4968):527-533.

55. Schatzl H, Gelderblom HR, Nitschko H, von der Helm K. Analysis of non-infectious HIV particles produced in presence of HIV proteinase inhibitor. Arch Virol. 1991;120(1-2):71-81.

56. Kaplan AH, et al. Partial inhibition of the human immunodeficiency virus type 1 protease results in aberrant virus assembly and the formation of noninfectious particles. J Virol. 1993;67(7):4050-4055.

57. Rosenbloom DI, Hill AL, Rabi SA, Siliciano RF, Nowak MA. Antiretroviral dynamics determines HIV evolution predicts therapy outcome. Nat Med. 2012;18(9):1378-1385.

58 . Kempf DJ, et al. Incidence of resistance in a double- blind study comparing lopinavir/ritonavir plus stavudine and lamivudine to nelfinavir plus stavudine and lamivudine. J Infect Dis. 2004;189(1):51-60.

59. Doyon L, Croteau G, Thibeault D, Poulin F, Pilote $\mathrm{L}$, Lamarre D. Second locus involved in human immunodeficiency virus type 1 resistance to protease inhibitors. J Virol. 1996;70(6):3763-3769.

60. Dam E, et al. Gag mutations strongly contribute to HIV-1 resistance to protease inhibitors in highly drug-experienced patients besides compensating for fitness loss. PLoS Pathog. 2009;5(3):e1000345.

61. Gatanaga $\mathrm{H}$, et al. Amino acid substitutions in gag protein at non-cleavage sites are indispensable for the development of a high multitude of HIV-1 resistance against protease inhibitors. J Biol Chem. 2002;277(8):5952-5961.

62. Gupta RK, Kohli A, McCormick AL, Towers GJ, Pillay D, Parry CM. Full-length HIV-1 gag determines protease inhibitor susceptibility within in vitro assays. AIDS. 2010;24(11):1651-1655.

63. Kolli M, Stawiski E, Chappey C, Schiffer CA. Human immunodeficiency virus type 1 proteasecorrelated cleavage site mutations enhance inhibitor resistance. J Virol. 2009;83(21):11027-11042.

64. Robinson LH, Myers RE, Snowden BW, Tisdale M, Blair ED. HIV type 1 protease cleavage site mutations and viral fitness: Implications for drug susceptibility phenotyping assays. AIDS Res Hum Retroviruses. 2000;16(12):1149-1156.

65. Parry CM, Kolli M, Myers RE, Cane PA, Schiffer C, Pillay D. Three residues in HIV-1 matrix contribute to protease inhibitor susceptibility and replication capacity. Antimicrob Agents Chemother. 2011;55(3):1106-1113.

66. Chojnacki J, et al. Maturation-dependent HIV-1 surface protein redistribution revealed by fluorescence nanoscopy. Science. 2012;338(6106):524-528.

67. Shan L, et al. A novel PCR assay for quantification of HIV-1 RNA. J Virol. 2013;87(11):6521-6525.

68. Pierson T, et al. Characterization of chemokine receptor utilization of viruses in the latent reservoir for human immunodeficiency virus type 1.J Virol. 2000;74(17):7824-7833.

69. Lengauer T, Sander O, Sierra S, Thielen A, Kaiser R. Bioinformatics prediction of HIV coreceptor usage. Nat Biotechnol. 2007;25(12):1407-1410. 